La Arquitectura de la Ciudad-Aldo Rossi / Bassett A., Castañeda A., Falcón L. , Sotelo Y../ abassetto11@hotmail.com, alvarojesusni@hotmail.com, luisefalcon24@gmail.com, andreinasotelo94@gmail.com

\section{La Arquitectura de la Ciudad- Aldo Rossi}

\section{Alyuska Octavia Bassett, Álvaro Jesús Cas- tañeda, Yosi Andreina Sotelo, Luis Enri- que Falcón Hernández/}

abassetto11@hotmail.com

alvarojesusni@hotmail.com

luisefalcon24@gmail.com

andreinasotelo94@gmail.com

Aldo Rossi nació en Milán en el año 1931 obtuvo el título de arquitecto por el politécnico Di Milano en 1959, primer arquitecto italiano en recibir el Premio Prinsker de Arquitectura en 1990 y precursor del modernismo. Fallece en 1997.

El Arquitecto Rossi fue el creador en 1966 de la Gran Obra "La arquitectura de la ciudad" en la que define a la Arquitectura como un TODO, haciendo estudio de ella, auxiliándose de distintas ciencias que estudian a la sociedad como la Antropología, psicología, el arte, entre otras; los que permite obtener distintos punto de vista de expertos de cada Rama.

"La Arquitectura es parte integrante del hombre en su construcción. La Arquitectura es la escena fija de las vicisitudes del hombre; con toda la carga de los sentimientos de las generaciones, de los acontecimientos públicos, de las tragedias privadas, de los hechos nuevos y antiguos..." nos dice Rossi.
El Pensamiento de Rossi era distinto a los demás escritores de arquitectura de la Época, ya que él consideraba que la arquitectura trascendía a través del tiempo, proporcionaba memoria histórica y era parte de la condición de vida del ser humano, el alegaba que la arquitectura provocaba recuerdos, emociones y sensaciones en la sociedad; en este libro analiza cada elemento que la compone con el objetivo de dar respuesta a esas problemáticas y que la ciudad ya mejorada sea un lugar que el ser humano pueda compartir y sentirse a gusto, manteniendo su historia y sobre todo pueda provocar distintas reacciones a sus ciudadanos.

Este historiador al igual que muchos le da la verdadera importancia que debe de tener la ciudad como obra Arquitectónica analizando cada elemento que la comLa arquitectura de la ciudad

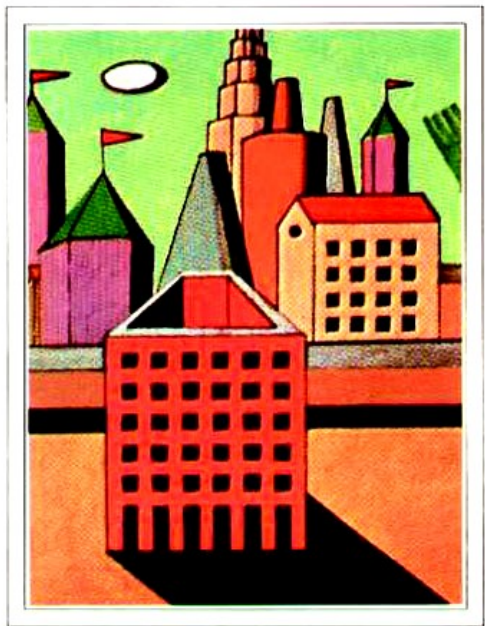
pone, inclinándose por el rescate de los espacios que forjen las relaciones sociales de convivencia, recreación e interacción entre los ciudadanos de la misma como espacios comunitarios, plazas y edificaciones específicas para actividades determinadas.

Para poder entender la Ciudad como un Todo, debemos estudiar a los creadores de la misma, debido a que fue diseñada con el objetico de satisfacer las necesidades de cada usuario, por lo antes expuesto es que Aldo Rossi analiza la ciudad haciendo uso de distintas ciencias, buscando la integración y la homogeneidad de la Arquitectura.

El crecimiento de la ciudad tiende más a la Evolución que a la conservación, a través de numerosos y varios momentos de formación, la agrupación de los mismos conforman la unidad urbana en su complejo, la ciudad es un todo y hay que analizarlo desde ese todo para luego llegar a pequeñas dimensiones. 
"He divido este libro en 4 partes; en la primera me ocupo de los problemas de descripción y de clasificación y, por lo tanto, de los problemas tipológicos; en la segunda, de la estructura de la ciudad por partes; en la tercera, de la arquitectura de la ciudad y de locus sobre el que esta persiste y, por lo tanto, de la historia urbana; en la cuarta en fin, aludo a las principales cuestiones de la Dinámica urbana y al problema de la política como elección.

La Comodidad de cualquier edificio comprende de tres objetos principales, su situación, su forma, la distribución de sus partes, esto representa tanto la imagen de una cosa que copiar o que imitar perfectamente, cuanto la idea de un elemento que debe servir como regla del modelo. Los Problemas tipológicos divididos en dos, la primera parte de la proposición, el autor descarta la posibilidad de algo que imitar "La Creación de un modelo, es decir no se haría arquitectura". La segunda afirma que la arquitectura "Modelo o forma" hay un elemento que tiene su propio papel.

Aldo Rossi en esta frase nos dice que cada obra arquitectónica debe poseer alguna característica o elemento que lo distinga por lo que no aprueba la posibilidad de imitar algo definiéndolo "No arquitectura", la originalidad del elemento tiende a poseer un grado de importancia con respecto a otro.

Es inconcebible pensar que los hechos urbanos cambian de alguna manera a causa de sus dimensiones. Relegar los problemas de la Ciudad a un problema de dimensiones, quiere decir entender que las soluciones están en la proyección al exterior del proceso de crecimiento, es decir, en la descentralización; tesis solución son ambos controvertidos.

La Modificación de la estructura territorial urbana que podemos seguir con absoluta precisión, a través de los mapas históricos catastrales indica el surgimiento de la burguesía urbana y el fenómeno de la concentración progresiva del capital.

No se puede ver a todo el conjunto urbano como un problema, sino que para buscar soluciones es necesario analizarlo sectorialmente, iniciando de micro a macro escala y así buscando la perfección y la solución de funcionabilidad de un conjunto urbano, llegan a construir la Ciudad perfecta con la que Aldo Rossi Soñaba.
Para estudiar a una ciudad, los planos urbanos antiguos son necesarios para delimitar el inicio de un conjunto urbano y a su vez la pérdida del mismo. Las Ciudades antiguas agrupadas en círculos concéntricos estaban ordenados en su centro la burguesía y la parte más lejana al centro las industrias, cosa que se ha revertido en la actualidad.

Para analizar la complejidad de los hechos urbanos, en otros palabras definir y clasificar, ya sea una calle, una ciudad, su función, su arquitectura y sucesivamente sus sistemas de calles posibles en la ciudad y otras muchas cosas tendremos que ocuparnos por lo tanto de la geografía urbana, la topografía urbana, de la arquitectura y otras disciplinas, los cuales no han sido objeto de trabajo sistemático en el campo de las ciencias urbanas.

La complejidad de los hechos urbanos representa un alto grado de análisis por parte de diferentes materias de estudio con el objetivo de llevar a cabo soluciones en cuanto a los muchos problemas que la ciudad presenta desde su estructura, morfología, conectividad y continuidad. En la actualidad no se le da importancia a todas estas ciencias y eso produce la ciudad que actualmente tenemos.

"También sin sus fábricas suntuosisimas la ciudad puede aparecer bella y respirar hermosura. Pero lo mismo es decir bella Ciudad que buena arquitectura..." 